Int. J. Dev. Biol. 60: 57-63 (2016)

doi: $10.1387 / \mathrm{ijdb} .1503811 \mathrm{~d}$

\title{
pdzrn3 is required for pronephros morphogenesis in Xenopus laevis
}

\author{
SILVIA MARRACCI, ALBERTO VANGELISTI, VITTORIA RAFFA, \\ MASSIMILIANO ANDREAZZOLI and LUCIANA DENTE* \\ Laboratory of Cell and Developmental Biology, Dept. of Biology, University of Pisa, Pisa, Italy
}

\begin{abstract}
Pdzrn3, a multidomain protein with E3-ubiquitin ligase activity, has been reported to play a role in myoblast and osteoblast differentiation and, more recently, in neuronal and endothelial cell development. The expression of the pdzrn3 gene is developmentally regulated in various vertebrate tissues, including muscular, neural and vascular system. Little is known about its expression during kidney development, although genetic polymorphisms and alterations around the human pdzrn3 chromosomal region have been found to be associated with renal cell carcinomas and other kidney diseases. We investigated the pdzrn3 spatio-temporal expression pattern in Xenopus laevis embryos by in situ hybridization. We focused our study on the development of the pronephros, which is the embryonic amphibian kidney, functionally similar to the most primitive nephric structures of human kidney. To explore the role of pdzrn3 during renal morphogenesis, we performed loss-of-function experiments, through antisense morpholino injections and analysed the morphants using specific pronephric markers. Dynamic pdzrn3 expression was observed in embryonic tissues, such as somites, brain, eye, blood islands, heart, liver and pronephros. Loss of function experiments resulted in specific alterations of pronephros development. In particular, at early stages, pdzrn3 depletion was associated with a reduction of the pronephros anlagen and later, with perturbations of the tubulogenesis, including deformation of the proximal tubules. Rescue experiments, in which mRNA of the zebrafish pdzrn3 orthologue was injected together with the morpholino, allowed recovery of the kidney phenotypes. These results underline the importance of pdzrn3 expression for correct nephrogenesis.
\end{abstract}

KEY WORDS: Pdzrn3, pronephros anlagen, tubulogenesis, Xenopus embryogenesis

\section{Introduction}

Pdzrn3 is a multitasking protein, containing a RING finger motif with E3-ubiquitin ligase activity and two PDZ binding domains, which mediate protein-protein interactions. Several protein ligands of Pdzrn3 have been identified in different tissues, suggesting specific roles in distinct transduction signalling. A direct interaction of Pdzrn3 with the receptor tyrosine kinase MuSK has been reported in murine neuromuscular junctions, showing a role in membrane receptor clustering (Lu et al., 2007). Perturbation of Pdzrn3 expression in cultured mesenchymal progenitor cells demonstrated its implication during myoblast and osteoblast differentiation, through negative regulation of Wnt signalling pathway (Ko et al., 2006; Honda et al., 2010). Expression of zebrafish pdzrn3 in neuronal tissues and binding of Pdzrn3 recombinant protein with Kidins220 suggested a role in retinal ganglion cell fasciculation and neurogen- esis (Dente et al., 2011; Andreazzoli et al., 2012). More recently, Pdzrn3-mediated ubiquitination of Dishvelled3 (Dvl3) was reported in murine endothelial cells and its requirement for mechanisms of vascular morphogenesis, mediated by Wnt/planar cell polarity signalling, was demonstrated (Sewduth et al., 2014).

The pdzrn3 gene is widely expressed in neuronal, skeletal and vascular tissues of vertebrates (Ko et al., 2006; Lu et al., 2007; Dente et al., 2011; Sewduth et al., 2014). Its expression in mouse kidney has been also reported, but little is known about its renal developmental regulation. On the other hand, several lines of evidence support correlations between human Pdzrn3 gene and different renal tumours and diseases. A possible involvement in

Abbreviations used in this paper: pdzrn3, PDZ-RING3; PDZ, PSD-95/Discs-large/ ZO1 domain; RING, really interesting new gene; st, stage.

\footnotetext{
*Address correspondence to: Luciana Dente. Laboratory of Cell and Developmental Biology, Dept. of Biology, University of Pisa, SS12 Abetone e Brennero, 56127 Pisa, Italy. Tel. +39-050-221-1488. Fax: + 39-050-221-1495. e-mail: luciana.dente@unipi.it
}

Accepted: 26 January 2016.

ISSN: Online 1696-3547, Print 0214-6282 
the regulation of sodium-dependent urate transport was firstly suggested, when a potential interaction between Pdzrn3 and the sodium-coupled monocarboxylate transporter, SMCT1, at the apical membrane of the renal proximal tubules was communicated (Anzai and Kimura, 2007). More recently, genomic rearrangements of the human pdzrn3 gene region on chromosome 3 have been associated to renal conventional carcinomas (RCC), characterized by unbalanced translocation $\mathrm{t}(3: 6)$ or $\mathrm{t}(3: 12)$ (Yusenko et al., 2010). The location of pdzrn3 gene in the human genome is just near the breakpoint at 3p12.3 position. In the kidney of a patient with unbalanced $\mathrm{t}(3: 12)$ translocation, a decreased pdzrn3 expression level was also documented (Yusenko et al., 2010). Furthermore, during a genome-wide screening of type2-diabetic patients with end-stage renal disease, a specific SNP polymorphism in the human pdzrn3 gene was associated with increased risk of death on dialysis (Murea et al., 2011). Other examples of associations between genetic alterations in the short arm of chromosome 3 and malformations of kidney and urinary tract (together with neurode-
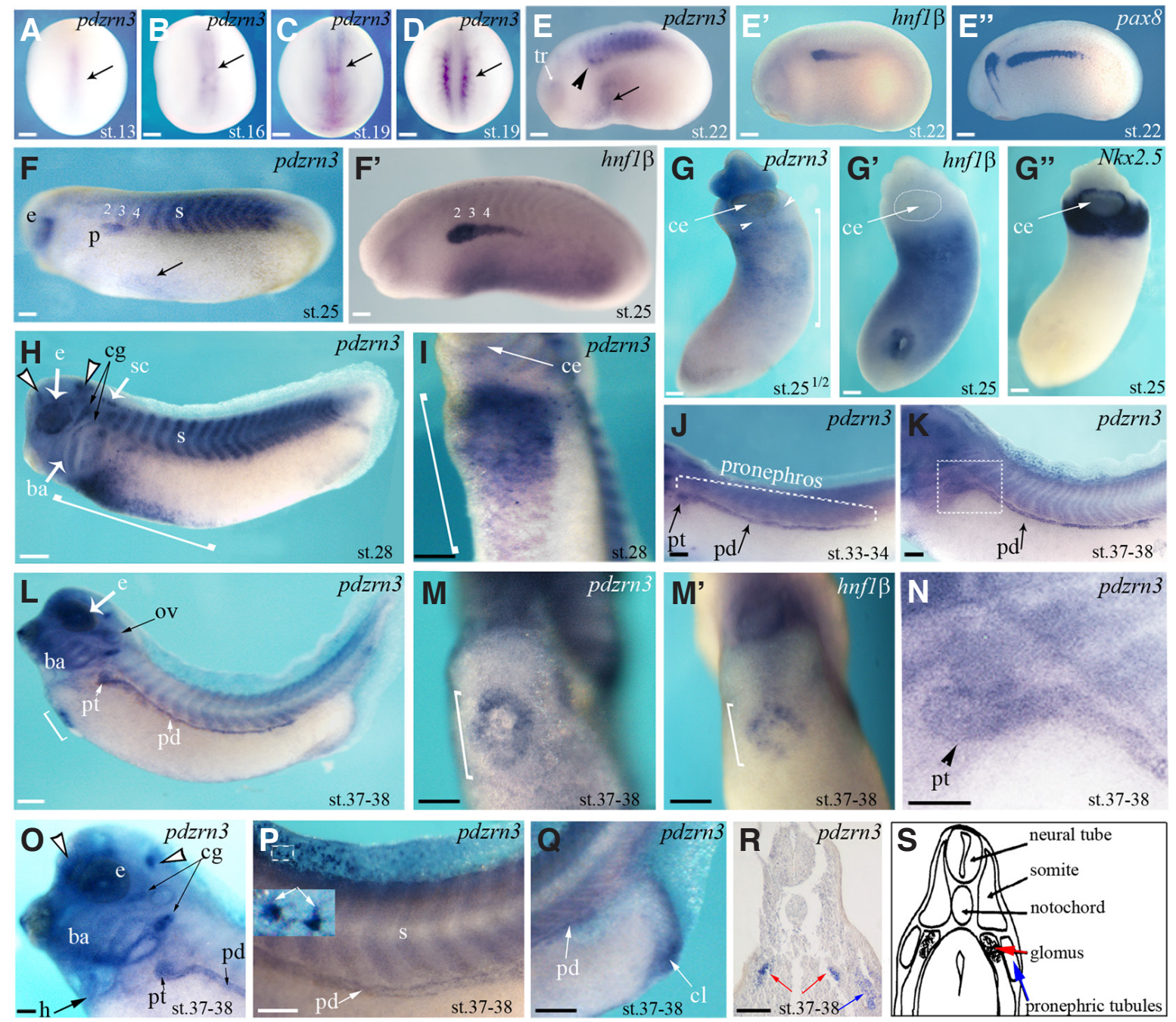

Fig. 1. Whole-mount in situ hybridization analysis of pdzrn3 during Xenopus laevis development. Nieuwkoop-Faber stages (st) of embryogenesis are indicated at the bottom right corner of each panel. The analysed genes are indicated at the top right corner of each panel. Abbreviations: branchial arches (ba), cement gland (ce), central nervous system (CNS), cloaca (cl), cranial ganglia (cg), eye vesicle (e), heart (h), otic vesicle (ov), pronephric anlagen (p), pronephric duct (pd), pronephric proximal tubules (pt), somites (s), spinal cord (sc), trigeminal anlagen (tr). (A,D) Dorsal views of pdzrn3 hybridized embryos during neurulation; black arrows indicate the specific pdzrn3 signal. (E-E") Comparison of embryos hybridized with pdzrn3, hnf1 $\beta$ and pax8 (lateral views, anterior left); black arrowhead in (E) underlines the pdzrn3 signal below the somites; black arrow indicates the specific pdzrn3 signal in the ventral region. (F,F') Comparison of embryos hybridized with pdzrn3 and hnf1 $\beta$. Somites numbers 2, 3, 4 are indicated and the ventral region is pointed by the arrow. (G-G") Ventral views of embryos hybridized with pdzrn3, hnf1 $\beta$ and Nkx2.5. In (G) the white bracket indicates the ventral region and white arrowheads point to a region below the ce gland. The ce is dashed in (G'). (H) Lateral view, anterior to the left and (I) ventral view (head to the top) showing the strong signal in the ventral region of embryos (white bracket); open arrowheads point to specific regions of the CNS. (J,K) Lateral views of the developing pronephros at st. 33-34 and 37-38. The dashed box in (K) corresponds to the enlarged view in panel (N). (L) Complete view of the embryo at st. 37-38. (M-Q) Details of specific domains of expression. White brackets in (L), (M) and (M') indicate the liver anlagen. (N) Enlargement of (K) dashed box. (O) Particular of the embryo head; open arrowheads: the regions of the CNS already detectable at st. 28. (P) Particular of the dorsal fin cells are highlighted in dashed box. (R) Transversal section of embryo, showing specific pdzrn3 signal in glomus (red arrows) and tubules (blue ar-

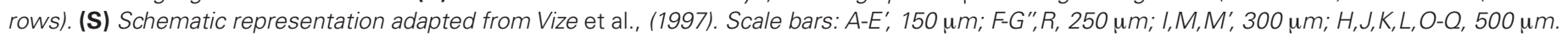


velopmental and intellectual disability) were reported in cases of $3 p$ interstitial deletions (Tutulan-Cunita et al., 2012).

To study the genes involved in vertebrate nephrogenesis, the amphibian Xenopus laevis has been often exploited as model system. Indeed, its embryonic kidney, the pronephros, is functionally similar to the most primitive nephric structures of human kidney, and it is easy to analyze its formation by manipulating the activity of involved genes (Hensey et al., 2002).

Here we have analysed the developmental expression pattern of pdzrn3 gene in Xenopus laevis embryos. We found that it is expressed in several mesodermal and neuroectodermal sites. In particular, it is present in the pronephric tubule anlagen, and at tadpole stages, when the pronephros is fully functional as excretory organ, also in glomus, tubules and collecting duct. To verify the need of pdzrn3 expression during Xenopus renal morphogenesis, we performed in vivo loss-of-function experiments mediated by morpholino antisense oligonucleotide. The clear alterations in the pronephros structures, induced by morpholino injections, suggest the involvement of $p d z r n 3$ in the mechanisms that regulate Xenopus laevis nephrogenesis.

\section{Results}

\section{Spatio-temporal expression pattern of pdzrn3 gene during Xenopus development}

The analysis of the expression pattern of pdzrn3 gene in Xenopus laevis embryos was performed by whole mount in situ hybridization, using as probe a DIG-labelled cRNA derived from full length Xenopus-pdzrn3 mRNA sequence (NCBI accession NM_001097041). The analysis revealed a dynamic pdzrn3 expression (Fig. 1). A specific signal is firstly detectable at stage 13 (early neurula) in dorsal medial line of the hybridized embryos (Fig. $1 \mathrm{~A}$, black arrow). At later neurula stages (st. 16 to 19) pdzrn3 is expressed in neural folds (Fig. 1 B-D, black arrows). At stage 22 (Fig. 1E) it is expressed in the developing somites, in a ventral region presumably corresponding to blood islands (black arrow) and in trigeminal anlagen (tr). pdzrn3 hybridization signal located below the somites 2-4 (1E, black arrowhead) partially overlaps the hybridization signal of two pronephric markers of the pronephros anlagen: $h n f 1 \beta$ (Fig. 1E') and pax8 (Fig. 1E'). pax8 is a pairedbox gene with an early crucial role in nephric lineage specification and later in tubulogenesis; $h n f 1 \beta$ encodes for a transcription factor involved in mesoderm induction and later in pronephros formation (Carrol and Vize, 1999; Vignali et al., 2000). In particular, hnf1 $\beta$ is expressed at high levels in the proximal tubules of Xenopus

Fig. 2. Analysis of pdzrn3 depletion effects using pax8 and hnf1 $\beta$ pronephric markers. Mo-pdzrn3 was injected into the marginal zone of one blastomere of Xenopus embryos at 2-cell stage; lacZ mRNA was used as lineage tracer. Control and injected sides of the same embryo are shown, respectively. Stages of embryos are indicated at the bottom right corner of each panel, while the analyzed gene is indicated to left of each row. Abbreviations: otic vesicles (ov), pronephric proximal tubules (pt). (A-F) Impairment of pdzrn3 gene function does affect early expression domains of pax8 and later the morphogenesis of nephrostomes, indicated by arrows. (G-L) In situ hybridization with hnf1 $\beta$ reveals reduction of the pronephron anlagen (black brackets) and pt alterations. The three nephrostomes, clearly detectable in the controls (arrowheads) are indistinct in the morphants. Embryos are shown in lateral views. Scale bars: $A, B, G, H$, $150 \mu \mathrm{m} ; C-F, I-L, 250 \mu \mathrm{m}$. pronephros (Desgrange and Cereghini, 2015). At stage 25 (Fig. $1 \mathrm{~F})$ pdzrn3 is expressed in eye vesicle (e) and in somites (s). The expression in the pronephric anlagen $(p)$ resembles that revealed with $h n f 1 \beta$ probe (compare Fig. $1 \mathrm{~F}$ and $1 \mathrm{~F}^{\prime}$ ). Moreover, the hybridized embryos show pdzrn3 expression in the ventral region (Fig. $1 \mathrm{~F}$, black arrow; Fig. $1 \mathrm{G}$ ventral view, white bracket). This region partially overlaps with the corresponding signal of $h n f 1 \beta$ in Fig. 1F' and Fig. 1G'). Furthermore, pdzrn3 expression was detected in the area located just below and around the cement gland (ce, white arrowheads). Hybridization with the heart anlagen marker Nkx2.5 showed partial overlapping of these domains (Fig. 1G").

At stage 28 (Fig. 1H, I ventral view), pdzrn3 expression is present in eye vesicle (e), cranial ganglia (cg), branchial arches (ba), somites (s), some regions of central nervous system (CNS) indicated by open arrowheads, spinal cord (sc) and ventral region

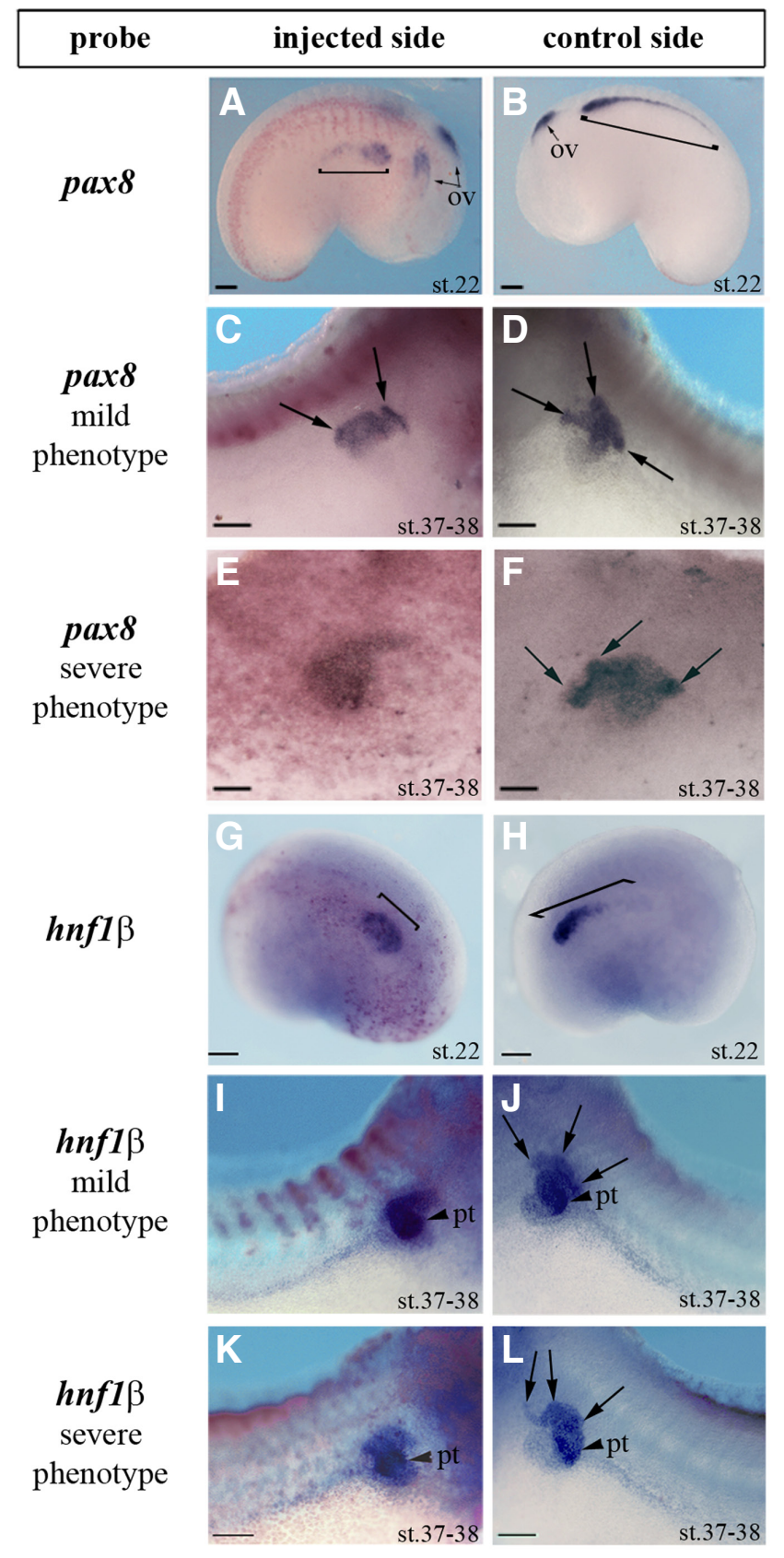


of embryos (white bracket).

At later developmental stages (st. 33-34 and 37-38) pdzrn3 expression is detectable in nephrostomes, pronephric tubules (pt) and in pronephric duct (pd) (Fig. $1 \mathrm{~J}, \mathrm{~K}, \mathrm{~L}, \mathrm{~N}-\mathrm{R})$. Furthermore, transverse sections of stage 37-38 hybridized embryos revealed specific expression also in the glomus (Fig. 1R, red arrows), as highlighted by the schematic representation (Fig. 1S). At stage 37$38, p d z r n 3$ signal is also detectable in other regions: liver anlagen, (Fig. 1L) as demonstrated by using $h n f 1 \beta$ as a marker (compare Fig. 1M and 1M' ventral views, white brackets); eye vesicle (e); some regions of CNS (open arrowheads); cranial ganglia (cg); branchial arches (ba); otic vesicle (ov) heart (h); dorsal fin cells (highlighted in dashed box); cloaca (cl) (Fig.1 L,O-Q).

\section{pdzrn3 depletion affects the early steps of pronephros development}

Taking into account the specific expression of pdzrn3 mRNA in the pronephros, we decided to verify the effects of its depletion during the different stages of Xenopus nephrogenesis. The

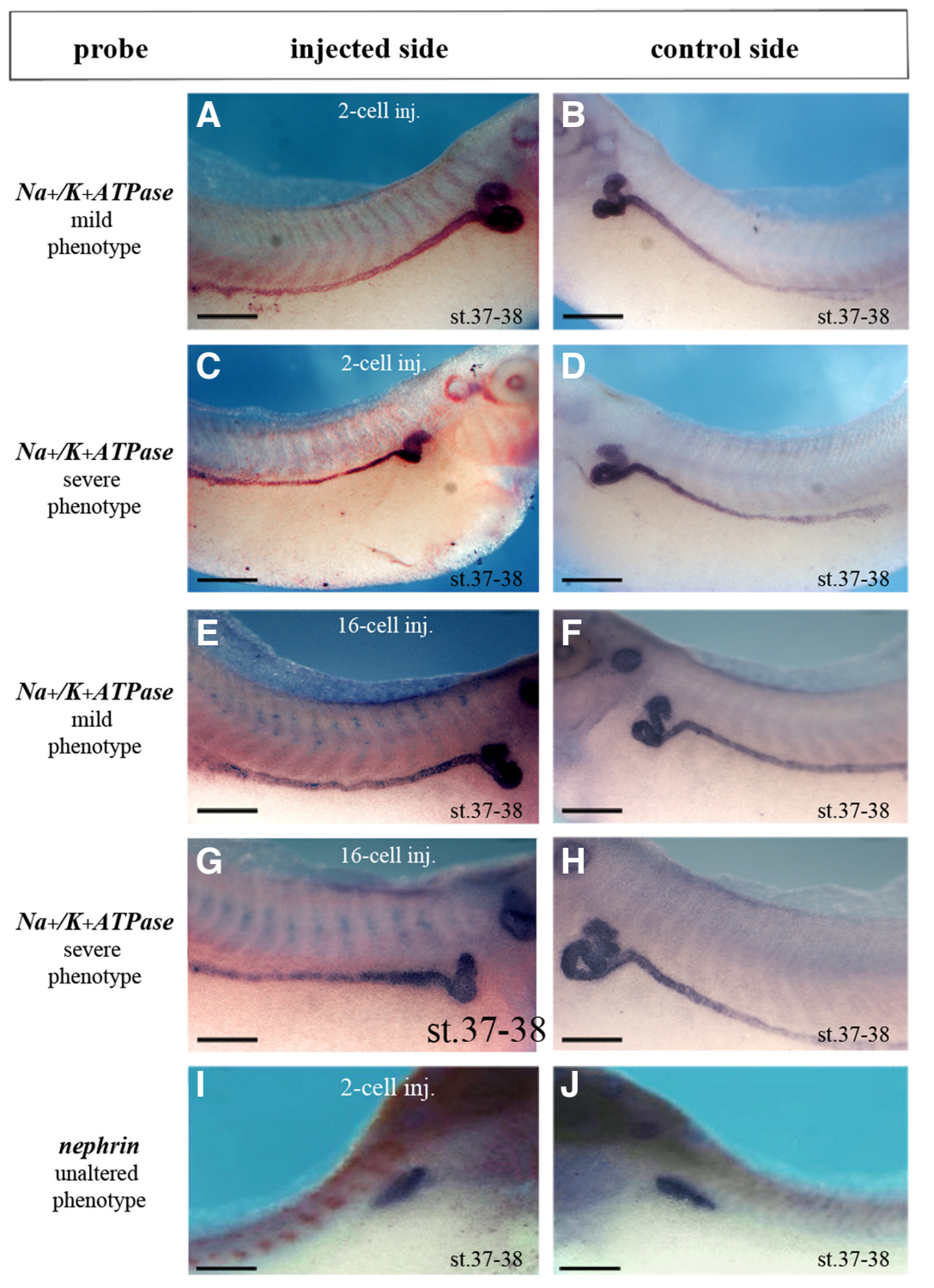

presumptive pronephros region, originating from the somitic intermediate mesoderm, starts to be specified at early neurula stage (st. 12.5); the pronephros anlagen is generated by cell condensation at late neurula (st. 22). The complete organ is functional at tadpole stage (st. 37-38) and consists of a glomus that filters into the coelum; the filtrate is reabsorbed by the nephrostomes and driven to the tubules and the duct (Vize et al., 1997; Brennan et al., 1999; Asashima et al., 2009; Desgrange and Cereghini, 2015).

We performed targeted loss of function experiments, by injecting Mo-pdzrn3, a specific antisense oligonucleotide morpholino complementary to a 5' sequence of Xenopus pdzrn3 mRNA, designed to inhibit its translation into protein. The standard Gene Tools Control-morpholino was used to control for non-specific responses. To target the presumptive mesoderm, Mo-pdzrn3 was injected into the marginal zone, a region located between the vegetal and animal hemispheres, of one blastomere of Xenopus embryos at 2-cell stage. $\beta$-galactosidase was used as a tracer to follow the distribution of the injected oligonucleotide. The uninjected side of the embryo was used as internal control of the experiments. The analysis of the morphants was performed by using different pronephric markers, in order to distinguish the distinct developing structures. pax 8 and $h n f 1 \beta$ are early expressed genes, involved in the initial patterning of the marginal zone mesoderm, but also implicated in later processes of kidney morphogenesis; $\mathrm{Na}^{+} / \mathrm{K}^{+}$ATPase and nephrin can be used as later pronephric markers that start to be expressed since tailbud stages 25 and 26 . In particular, $\mathrm{Na}^{+} / \mathrm{K}^{+}$ATPase encodes for a key pump enzyme, expressed at level of tubules and duct; nephrin encodes for a transmembrane protein that is expressed at level of the glomus (Eid and Brändli, 2001; Gerth et al., 2005).

Comparison between the Mo-pdzrn3 injected side and the control side of embryos hybridized with pax8 probe, revealed significant difference in the renal expression domains either at early (st. 22) or at later (st. 37-38) analysed stages. In particular $80.6 \%$ of the morphants $(n=36)$ showed an altered expression of pax8 domain at st. 22 (Fig. 2 A,B, black brackets). At st. 37-38, morphants hybridized with pax8 showed a phenotype $(65.7 \%, \mathrm{n}=35)$ with an irregular positioning (indicated by black arrows) of the nephrostomes ("mild" phenotype) (Fig. 2 C, D). Fewer embryos showed a "severe" phenotype, with a strongly reduced pax8 expression (Fig. 2 E,F).

Similar results were obtained by using the pronephric marker hnf1 $\beta$, which highlights very well the

Fig. 3. Analysis of pdzrn3 depletion effects using $\mathrm{Na}^{+} /$ $\boldsymbol{K}^{+} \boldsymbol{A T P a s e}$ and nephrin pronephric markers. Xenopus embryos were injected with Mo-pdzrn3 at 2-cell stage into the marginal zone of one blastomere or at 16-cell stage into V1.1 blastomere, as indicated at the top of the injected side panels. In situ hybridization with $\mathrm{Na}+/ \mathrm{K}+$ ATPase probe on embryos at st. 37-38(A-H) reveals that impairment of pdzrn3 gene function does affect the formation of pronephric proximal tubules (pt) but not the formation of the duct $(\mathbf{A}, \mathbf{C}, \mathbf{E}, \mathbf{G})$, or the formation of the glomus, revealed by in situ hybridization with nephrin probe (I,J). Scale bars: A, J, $300 \mu \mathrm{m}$. 
pronephric proximal tubules (pt), while is poorly expressed in the duct (Desgrange and Casiraghi, 2015). In particular, $82 \%$ of the Mo-pdzrn3injected embryos $(\mathrm{n}=39)$ showed a reduced expression domain of $h n f 1 \beta$ at st. 22 (Fig. $2 \mathrm{G}-\mathrm{H}$, white brackets). $71.8 \%$ of injected embryos $(n=39)$ showed alterations in the coiling and/ or formation of pt convolutions at st. $37-38$. In particular, $60.7 \%$ displayed a "mild" phenotype (lighter alterations) (Fig. 2 I,J), and $39.36 \%$ a "severe" phenotype (stronger alterations) (Fig. 2 K,L).

Hybridization on embryos at st.37-38 with the late marker $\mathrm{Na}^{+} /$ $K^{+}$ATPase revealed normal development of the duct, but an altered pt coiling in $81.8 \%$ of Mo-pdzrn3 injected embryos $(n=33): 60 \%$ exhibited a mild phenotype (weaker alteration of pt coiling; Fig. $3 \mathrm{~A}, \mathrm{~B}$ ); $40 \%$ a severe phenotype (drastic alteration of pt coiling; Fig. 3 C,D). To better restrict the field of the morpholino effects, we also verified the consequence of injecting Mo-pdzrn3 at 16cell stage into V1.1 blastomere, which is more specifically fated to form the pronephric anlagen. $77.2 \%$ of the embryos injected at 16-cell stage $(n=35)$ and analysed at st.37-38 with $\mathrm{Na}^{+} / \mathrm{K}^{+}$ATPase probe, presented alteration of pt coiling: $74 \%$ displayed a "mild" phenotype (Fig. 3 E,F) and 26\% a "severe" phenotype (Fig. 3 G,H). In contrast, hybridization with nephrin probe that allows specific detection of the glomus did not reveal substantial alterations in the injected embryos (Fig. $3 \mathrm{I}, \mathrm{J}$ ).

All these results indicate that perturbation of pdzrn3 gene expression affects the determination of the pronephros field, mainly influencing morphogenesis and coiling of proximal tubules, whereas it is less effective on glomus and duct formation.

\section{Rescue experiments}

To test the specificity of the observed phenotypes, we designed a "rescue" experiment, using a full length cDNA of Danio rerio: $d$-pdzrn3 (accession n. JN108761) we cloned in a previous study (Dente et al., 2011). The zebrafish Pdzrn3 protein is very similar to the orthologous protein of Xenopus (sharing 70\% homology), whereas the differences between the two RNA are higher, in particular at the level of the 5' sequence corresponding to the morpholino Mo-pdzrn3 target (see Mat. and Met.). Therefore, we decided to inject together with Mo-pdzrn3, the full-length $d$-pdzrn3 RNA, in order to assess whether this may restore a normal phenotype. Firstly, different concentrations of in vitro transcribed $d$-pdzrn3 RNA (200, 300, 600 pg) were tested to verify the effects of its expression after injection in the marginal zone of one blastomere at two-cell stage. Higher concentrations of $d$-pdzrn3 RNA were toxic for the embryos, while the lower concentration (200pg) was safe and did not induce any alteration in the injected embryos. Following coinjection with Mo-pdzrn3, the phenotype of the pronephric tubules was

Fig. 4. Coinjections of Mo-pdzrn3 + full-length pdzrn3RNA of Danio rerio allow to significantly recover wild type phenotypes. The coinjections were carried out into one blastomere at 2-cellstage. Comparison of the injected and control side of Xenopus embryos reveals the rescue of the expression domains of pax8 $(\mathbf{C}, \mathbf{D})$ and $\operatorname{hnf} 1 \beta(\mathbf{G}, \mathbf{H})$ at stage 22, and correct formation of the pronephric tubules and of the nephrostomes (arrows) at stage 37-38, as revealed using $\mathrm{Na}+/ \mathrm{K}+$ ATPase probe (A,B), pax8 probe (E,F), and hnf1 $\beta$ (I,J). Scale bars: $A, B, E, F, I, J, 300 \mu \mathrm{m} ; C, D, G, H, 600 \mu \mathrm{m}$. efficiently rescued in a high percentage of the injected embryos hybridized with the pronephric markers at different stages (Fig. 4). We found $69.65 \%$ of rescue in $\mathrm{Na}^{+} / \mathrm{K}^{+}$ATPase st. 37-38 hybridized embryos, $n=51$ (Fig. 4 A,B); $69.65 \%$ of rescue in pax8 st. 22 embryos, $n=51$ (Fig.4 C,D); $69.7 \%$ of rescue in pax8 st. 37-38 embryos, $\mathrm{n}=43$ (Fig. $4 \mathrm{E}, \mathrm{F}$ ); $63 \%$ of rescue in $h n f 1 b$ st. 22 embryos, $n=35$ (Fig. 4 G,H); $68 \%$ of rescue in $h n f 1 \beta$ st. 37-38 embryos, $n=50$ (Fig. 4 I,J).

\section{Discussion}

The kidney is an excretory organ, able to dispose soluble waste substances out of the blood, while absorbing and recovering useful substances. Fishes and amphibians early develop a simple renal system, the pronephros, which is the functional filtering organ at larval stage. In Xenopus the pronephros represents a single nephron, corresponding to the basic unit of the mammalian adult kidney. It operates with essentially the same regulatory elements and the specific transcription factors that are needed for the nephrogenesis of higher vertebrate. Therefore, the definition of the pronephros regulator is also useful to understand the morphogenetic potential of mutated factors associated with kidney human diseases. We observed a dynamic expression of pdzrn3 mRNA since early developmental stages in

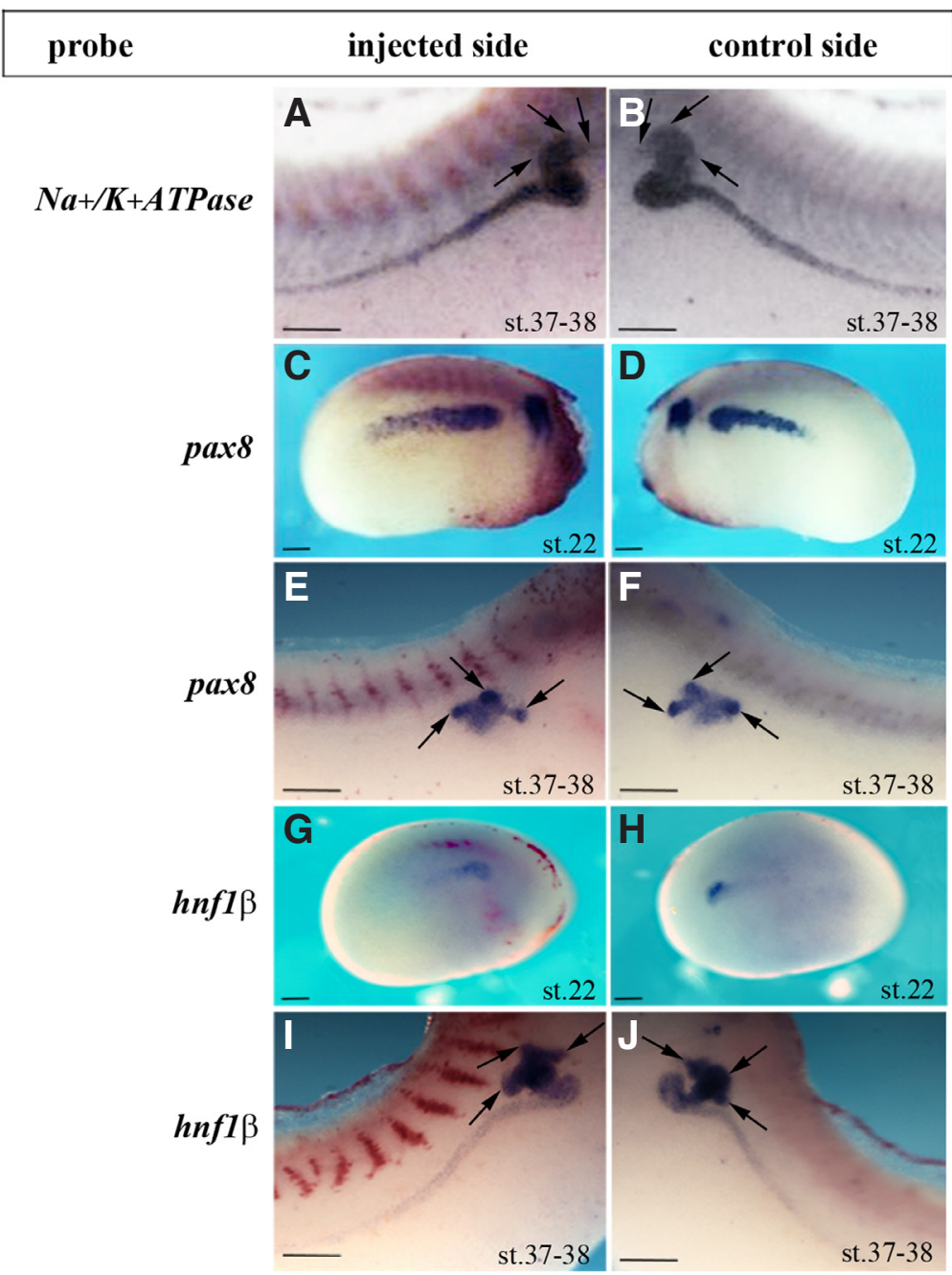


the pronephric tubule anlagen and later, in vascular and epithelial structures of the functional organ, therefore to verify whether pdzrn3 is involved in mechanisms of pronephros differentiation, we performed morpholino-mediated loss of function experiments. Antisense morpholino-oligonucleotides are typically exploited for functional studies in non-genetic model organisms, such as Xenopus. Nevertheless, their use in zebrafish and a recent comparison between morphants and mutants phenotypes has solicited some concerns about their specificity effects (Kok et al., 2015). In contrast, leading expert scientists, Blum and co-authors have recently reviewed the many advantages afforded by morpholinos in generic loss of function approaches, in particular in Xenopus animal model, but also in zebrafish, showing multiple examples of confirming results (Blum et al., 2015).

We found that perturbation of $p d z r n 3$ expression induced alterations in the pronephros anlagen formation, suggesting a role in the early steps of mesodermal differentiation at late gastrula stage that will be interesting to further investigate. Later in development, we detected in the injected side anomalies in the morphogenesis of the three nephrostomes and in the coiling of the proximal tubules, but not in the glomus or in the duct. The specificity of such observations was confirmed by rescue experiments, in which the orthologue $d$-pdzrn3RNA could restore normal phenotypes. It is not surprising to discover different effects of pdzrn3 depletion in distinct kidney structures. From the late neurula stage, the cells of the pronephros anlagen undergo distinct remodeling, oriented divisions, and alignment to constitute glomus, tubules and duct. Actually the pdzrn3gene is expressed in all three structures, where it could interact with different partners, possibly exerting different functions. Pdzrn3 protein contains a Ring finger-domain with E3ubiquitin ligase activity, two PDZ domains, a PDZ-binding motif at the carboxy-terminus and therefore may interact with multiple and diverse ligands.

Recently, it has been shown that murine Pdzrn3 directly interacts with Disheveled (Dvl3), inducing its ubiquitinization and favouring the endocytosis of the Frizzled/Dvl3-complex during mechanisms of vascular patterning (Sewduth et al., 2014). We speculate that Pdzrn3 could play a similar role in mechanisms of directional and coordinated cell extension for remodeling and coiling the pronephric tubules. The molecular mechanisms, required for correct tubulogenesis in Vertebrates, are becoming increasingly clear. The contribution of Wnt proteins in the induction of kidney mesenchyme has been proved, and some specific cues and signal transducers that are needed to organize and position the developing tubules, with a defined geometry, have been identified (Dressler 2006; McNeill 2009). The non-canonical Wnt/planar cell polarity (PCP) pathway has a crucial role in regulating migration and proper alignment of the dividing cells. Indeed, defects in the PCP signalling have been associated to severe renal diseases in human (Fisher et al., 2006; McNeill 2009). In the non-canonical pathway, the binding of Wnt to its receptor Frizzled, induces activation of Dvl, JNK and Rho family GTPases, promoting asymmetric cytoskeletal organization and convergent extension movements of the cells.

The involvement of Pdzrn3 in Wnt regulated pathway has been proposed also in other systems. Pdzrn3 can play an inhibitory role in the differentiation of $\mathrm{C} 2 \mathrm{C} 12$ myoblast cells into osteoblasts, through attenuation of the Wnt/beta-catenin canonical pathway (Honda et al., 2010).

Taken together all these information suggest that Pdzrn3 mediates diverse developmental processes. These include the early patterning of the marginal zone mesoderm that induces the formation of pronephric anlagen and later processes required for correct coiling of proximal tubular loops. Further studies are needed to identify correlated Pdzrn3 interactions and understand the corresponding functions.

\section{Materials and Methods}

\section{Plasmid constructions and in situ hybridization}

Xenopus embryos were staged according to Nieuwkoop and Faber (1967).

Whole-mount in situ hybridization was performed according to standard Harland's protocols with minor modifications (see D'Autilia et al., 2010; Marracci et al., 2013).

The cDNA clone, used to prepare in vitro transcribed $p d z r n 3$ probe for in situ hybridization, was generated by RT-PCR of mRNA extracted from stage 27 embryos. Specific primers: (Forward:5'-CACTCCAACGGGCACCGACCATGGGCTTTG-3'and (Reverse:5'-AGATAGTCGTGAATCCAGTCCTGCCAGGTACCATCTCCCT-3') were designed on the basis of the full length pdzrn3sequence of Xenopus laevis (NCBI accession no. NM_001097041). The amplified fragment was cloned in pGEM-T-easy plasmid and its identity to clone NM_001097041 was assessed by sequencing. To prepare sense and antisense probes, standard cRNA synthesis from linearized plasmids with SP6 and T7 RNA polymerases, was carried out, using a digoxigenin (DIG) labelling kit (Roche) according to manufacturer's recommendations.

Probes were transcribed from cDNA clones obtained from Peter Vize (pax8 and nephrin), André Brändli $\left(\mathrm{Na}^{+} / \mathrm{K}^{+}\right.$ATPase) and Robert Vignali ( $h n f 1 \beta)$. Sense transcripts were used as negative control (data not shown).

\section{Paraffin sectioning}

Whole-mount hybridized embryos were paraffin-embedded and cut with a Reichert-Jung Autocut 2040 Microtome. After Hoechst-counterstaining, the sections were washed and mounted in Aqua Poly/Mount (Polysciences Inc.) (see Marracci et al., 2015).

\section{Morpholino microinjections and rescue experiments}

A specific antisense morpholino oligonucleotide (Mo-pdzrn3) was designed against the 5' sequence upstream to the Pdzrn3 start codon of translation:

5'-CCAGTTCAAAGCCCATGGTCGGTGC-3'. GenBank searches failed to detect significant homologies of Mo-pdzrn3 elsewhere in the Xenopus genome. The Gene Tools Mo-standard (5'-CCTCTTACCTCAGTTACAATTTATA -3') was used for control morpholino injections.

Microinjections were performed as described previously (Lunardi et al., 2006). One picomole of Mo-standard control or Mo-pdzrn3 morpholino oligos were injected at the marginal zone of one blastomere into two cell stage embryos. $200 \mathrm{pg}$ of capped LacZ RNA was co-injected to visualize the injected side of the embryos.

For rescue experiments a Danio rerio pdzrn3 cDNA clone (GenBank accession no. JN108761) was used to prepare a synthetic capped mRNA by in vitro transcription, as described in Djiane et al., (2000).

$$
\begin{aligned}
& \text { MoPdzrn3_XI: 3' CGTGGCTGGTACCCGAAACTTGACC }{ }^{\prime}
\end{aligned}
$$

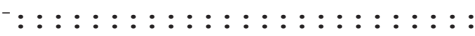

$$
\begin{aligned}
& \text { RNA pdzrn3_XI: }{ }^{\prime} \cdot \cdot \text { GCACCGACCAUGGGCUUUGAACUGG }{ }^{\prime}{ }_{3} \\
& \text { RNA pazrn3_Dr: }{ }^{5} \cdot \cdots \text { UAAUAAUA-AUGGGAUUUGAGUUGG }{ }^{\prime}{ }^{\prime} \text {, }
\end{aligned}
$$

\section{Acknowledgements}

We are grateful to Peter Vize, André Brändli and Robert Vignali for generously providing Xenopus pronephros markers. We thank Marzia Fabbri, Donatella De Matienzo and Elena Landi for technical assistance and Salvatore Di Maria for frog care. 


\section{References}

ANDREAZZOLI M, GESTRI G, LANDI E, D'ORSI B, BARILARI M, IERVOLINO A, VITIELLO M, WILSON SW, DENTE L (2012). Kidins220/ARMS interacts with Pdzrn3, a protein containing multiple binding domains. Biochimie 94: 2054-2057.

ANZAI N, KIMURA T (2007). Effect of the interaction between a novel PDZ protein and $\mathrm{Na}^{+}$dependent lactate transporter SMCT1 on renal urate transport. The Salt Science Research Foundation report -on line http://www.saltscience.or.jp/ general_research/2007/200721.pdf (Abstr. n. 0721: 48).

ASASHIMA M, ITO Y, CHAN T, MICHIUE T, NAKANISHI M, SUZUKI K, HITACHI K, OKABAYASHI K, KONDOW A, ARIIZUMI T (2009). In vitro Organogenesis from Undifferentiated Cells in Xenopus. Dev Dynam 238:1309-1320.

BLUM M, DE ROBERTIS E M, WALLINGFORD J B, NIEHRS C (2015). Morpholinos: Antisense and Sensibility. Dev Cell 35: 145-149.

BRENNAN H C, NIJJAR S, JONES E A (1999). The specification and growth factor inducibility of the pronephric glomus in Xenopus laevis. Development 126: 5847-5856.

CARROLL T J, VIZE P D (1999). Synergism between Pax-8 e Lim-1 in embryonic kidney development. Dev Biol 214: 46-59.

D'AUTILIA S, BROCCOLI V, BARSACCHI G, ANDREAZZOLI M (2010). Xenopus Bsx links daily cell cycle rhythms and pineal photoreceptor fate. Proc Natl Acad Sci USA 107: 6352-6357.

DENTE L, GESTRI G, TSANG M, KUDOH T, WILSON S W, DAWID I B, ANDREAZZOLI M (2011). Cloning and developmental expression of zebrafish pdzrn3. Int J Dev Biol. 55 (10-12): 989-993.

DESGRANGEA, CEREGHINIS (2015). Nephron Patterning: Lessons from Xenopus, Zebrafish, and Mouse Studies. Review. Cells 4: 483-499.

DRESSLER G R (2006) The Cellular Basis of Kidney Development Ann Rev Cell Dev Biol 22: 509-529.

DJIANE A, RIOU J, UMBHAUER M, BOUCAUT J, SHI D (2000). Role of frizzled 7 in the regulation of convergent extension movements during gastrulation in Xenopus laevis. Development 127: 3091-3100.

EID S R, BRÄNDLI A W (2001). Xenopus Na,K-ATPase: primary sequence of the beta2 subunit and in situ localization of alpha1, beta1, and gamma expression during pronephric kidney development. Differentiation 68: 115-125.

FISCHER E, LEGUE E, DOYEN A, NATO F, NICOLAS J F, TORRES V, YANIV M, PONTOGLIO M (2006). Defective planar cell polarity in polycystic kidney disease. Nat Genet 38: 21-23.

GERTH V E, ZHOU X, VIZE P D (2005). Nephrin expression and three-dimensional morphogenesis of the Xenopus pronephric glomus. Dev Dyn 233: 1131-1139.

HENSEY C, DOLAN V, BRADY H (2002). The Xenopus pronephros as a model system for the study of kidney development and pathophysiology. Nephrol Dial Transp/ 17: 73-74.

HONDA T, YAMAMOTO H, ISHII A, INUI M (2010). PDZRN3 negatively regulates
BMP-2-induced osteoblast differentiation through inhibition of Wnt signaling. Mol Biol Cell 21: 3269-3277.

KOK F O, SHIN M, NI C-W, GUPTA A, GROSSE A S, VAN IMPEL A, KIRCHMAIER B C, PETERSON-MADURO J, KOURKOULIS G, MALE I, DESANTIS D F, SHEPPARD-TINDELL S, EBARASI L, BETSHOLTZ C, SCHULTE-MERKER S, WOLFE S A, LAWSON N D (2015). Reverse genetic screening reveals poor correlation between morpholino-induced and mutant phenotypes in zebrafish. Dev Cell 32: 97-108.

KO J A, KIMURA Y, MATSUURA K, YAMAMOTO H, GONDO T, INUI M (2006). PDZRN3 (LNX3, SEMCAP3) is required for the differentiation of C2C12 myoblasts into myotubes. J Cell Sci 119(Pt 24): 5106-5113.

LU Z, JE H S, YOUNG P, GROSS J, LU B, FENG G (2007). Regulation of synaptic growth and maturation by a synapse-associated E3 ubiquitin ligase at the neuromuscular junction. J Cell Biol 177: 1077-1089.

LUNARDI A, CREMISI F, DENTE L (2006). Dystroglycan is required for proper retinal layering. Dev Biol 290: 411-420.

MARRACCI S, GIANNINI M, VITIELLO M, ANDREAZZOLI M, DENTE L (2013). Kidins220/ARMS is dynamically expressed during Xenopus laevis development. Int J Dev Biol 57 (9-10): 787-792.

MARRACCI S, MARTINI D, GIANNACCINI M, GIUDETTI G, DENTE L, ANDREAZZOLI M (2015). Comparative expression analysis of pfdn6a and tcp1 $\alpha$ during Xenopus development. Int J Dev Biol 59: 235-240.

MCNEILLH (2009). Planar cell polarity and the kidney. JAm Soc Nephrol20:2104-2111.

MUREA M, LU L, MA L, HICKS P J, DIVERS J, MCDONOUGH C W, LANGEFELD C D, BOWDEN D W, FREEDMAN B I (2011). Genome-wide association scan for survival on dialysis in African-Americans with type 2 diabetes. Am J Nephrol 33: 502-509.

NIEUWKOOP P D, FABER J (1967). Normal Table of Xenopus laevis. II edition. Amsterdam, The Netherlands: North Holland Publishing Company.

SEWDUTH RN, JASPARD-VINASSAB, PEGHAIRE C, GUILLABERT A, FRANZL N, LARRIEU-LAHARGUEF, MOREAU C, FRUTTIGERM, DUFOURCQP, COUFFINHAL T, DUPLÀA C (2014). The ubiquitin ligase PDZRN3 is required for vascular morphogenesis through Wnt/planar cell polarity signalling. Nat Commun 5: 1-13.

TUTULAN-CUNITA A C, PAPUC S M, ARGHIR A, ROTZER K M, DESHPANDE C, LUNGEANU A., BUDISTENAU M (2012). 3p interstitial deletion: novel case report and review. J Child Neurol 1062: 2012-2027.

VIGNALI R, POGGI L, MADEDDU F, BARSACCHI G (2000). hnf1 $\beta$ is required for mesoderm induction in the Xenopus embryo. Development 127: 1455-1465.

VIZE P D, SEUFERT D W, CARROLL T J, WALLINGFORD J B (1997). Model systems for the study of kidney development: use of the pronephros in the analysis of organ induction and patterning. Rev. Dev. Biol. 188: 189-204.

YUSENKO M V, NAGY A, KOVACS G (2010). Molecular analysis of germline $t(3 ; 6)$ and $t(3 ; 12)$ associated with conventional renal cell carcinomas indicates their rate-limiting role and supports the three-hit model of carcinogenesis. Cancer Genet Cytogenet 201: 15-23. 
Embryonic expression of Xenopus SGLT-1L, a novel member of the solute carrier family $\mathbf{5}$ (SLC5), is confined to tubules of the pronephric kidney

Samer R Eid, Anne Terrettaz, Katsumi Nagata and André W Brändli

Int. J. Dev. Biol. (2002) 46: 177-184

http://dx.doi.org/10.1387/ijdb.11902681

Cloning and developmental expression of zebrafish pdzrn3

Dente L, Gestri G, Tsang M, Kudoh T, Wilson S W, Dawid I B, Andreazzoli M

Int. J. Dev. Biol. (2011) 55:989-93.

http://dx.doi.org/10.1387/ijdb.113437ld

The role of angiogenic growth factors in organogenesis

Enrico Crivellato

Int. J. Dev. Biol. (2011) 55: 365-375

http://dx.doi.org/10.1387/ijdb.103214ec

Kidins220/ARMS is dynamically expressed during Xenopus laevis development Marracci S, Giannini M, Vitiello M, Andreazzoli M, Dente L

Int. J. Dev. Biol. (2013) 57: 787-792

http://dx.doi.org/10.1387/ijdb.130080sm

Comparative expression analysis of pfdn6a and tcp1 $\alpha$ during Xenopus development Marracci S, Martini D, Giannaccini M, Giudetti G, Dente L, Andreazzoli M Int. J. Dev. Biol. (2015) 59: 235-40

http://dx.doi.org/10.1387/ijdb.140275ma
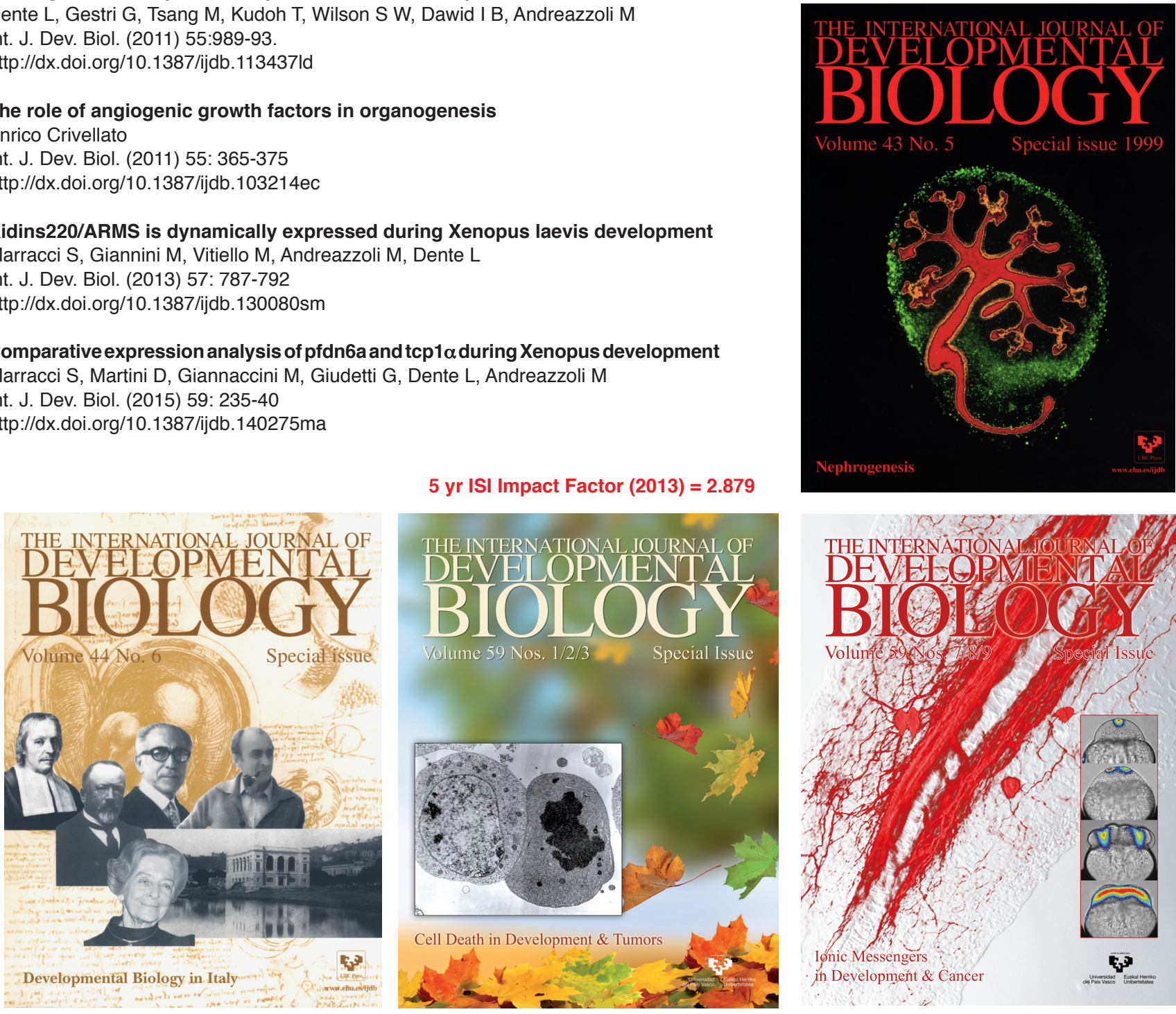

5 yr ISI Impact Factor $(2013)=2.879$
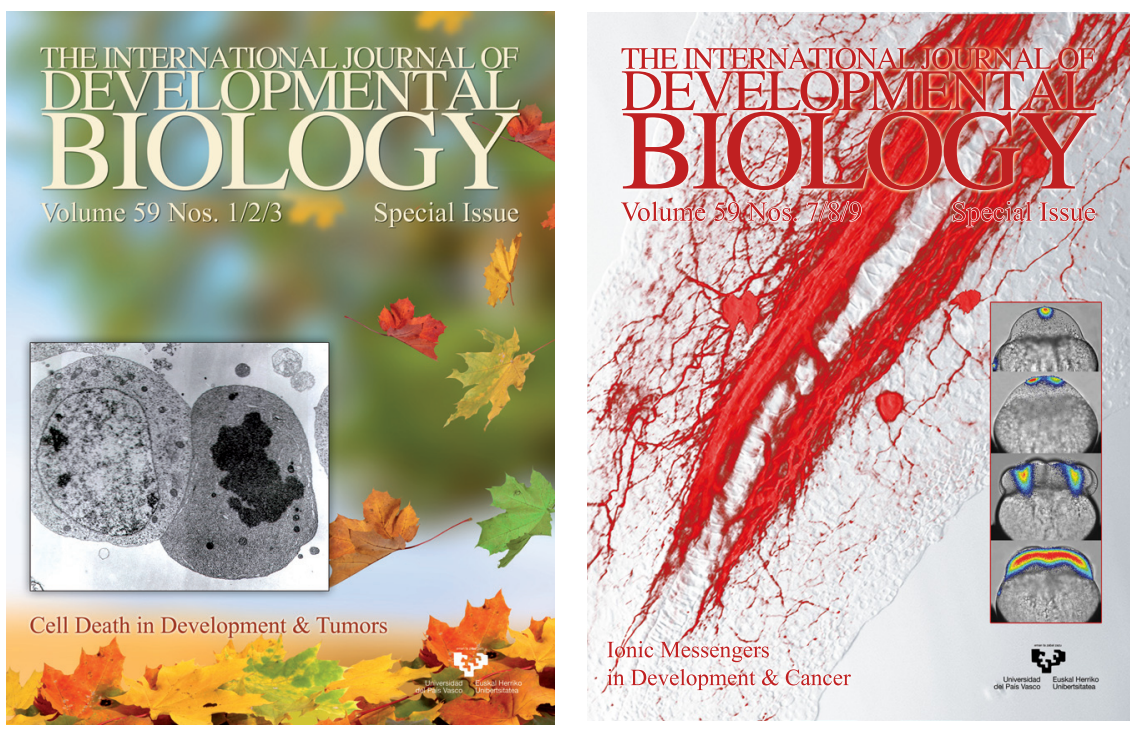